\title{
Avaliação da resiliência socioecológica como ferramenta para a gestão da fronteira amazônica: experiências e reflexões
}

\section{Social-ecological resilience assessment as a tool for management of the Amazon frontier: experiences and reflections}

\author{
Simone Athayde ${ }^{a}$ \\ Paula Bernasconi ${ }^{\text {b }}$ \\ Wendy-Lin Bartels ${ }^{c}$ \\ Rosane D. R. Seluchinesk ${ }^{d}$ \\ Robert Buschbacher ${ }^{\mathrm{e}}$ \\ ${ }^{a}$ Tropical Conservation and Development Program, \\ University of Florida, Gainesville, FL, USA \\ End. Eletrônico: simonea@ufl.edu

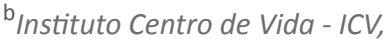 \\ Cuiabá, MT, Brasil \\ End.Eletrônico:paulabernas@gmail.com \\ 'School of Forest Resources and Conservation, Research Assistant Scientist, \\ University of Florida, Gainesville, FL, USA \\ End.Eletrônico: wendylin@ufl.edu \\ ¿Universidade do Estado de Mato Grosso - UNEMAT, \\ Alta Floresta, MT, Brasil \\ End. Eletrônico: rosane.rosa@unemat.br \\ ${ }^{e}$ Amazon Conservation Leadership Initiative, \\ University of Florida, Gainesville, FL, USA \\ End. Eletrônico: rbusch@ufl.edu
}

doi:10.18472/SustDeb.v7n2.2016.19936

A Amazônia brasileira compreende um conjunto único de paisagens e dinâmicas socioecológicas interdependentes, configuradas por uma diversidade de atores, ecossistemas, usos da terra e práticas de manejo de recursos naturais. No entanto, a sociedade brasileira e global vivencia um momento de alto risco em relação à sustentabilidade da mega biodiversidade das paisagens amazônicas. A transformação e degradação destes ambientes decorre do modelo de desenvolvimento adotado no país, em conjunto com o modelo político e fatores associados como mudanças climáticas, desmatamento, uso intensivo da terra para agropecuária, mineração, implementação de infraestrutura de forma inadequada, contaminação por agrotóxicos, entre outros. Estaremos à beira de um limiar (em inglês, "threshold") de transformação dos sistemas socioecológicos amazônicos, para ambientes mais secos, menos diversos e mais vulneráveis ao fogo, inundações, insegurança alimentar etc.? Como estabelecer um processo de governança para a Amazônia, considerando e respeitando a sua socio-biodiversidade, neste cenário de transformação? 
A noção de sistema socioecológico pode contribuir para o entendimento de uma região onde escalas geográficas e temporais, atores sociais, fatores econômicos e condições ambientais interagem em cadeia. O sistema ecológico compreende escalas que vão desde a unidade de recurso em estudo (como por exemplo, o gado, o milho, a castanha) até as comunidades ou ambientes nos quais se inserem, como o ecossistema, o bioma, a biosfera planetária. O sistema social compreende os atores sociais e suas instituições, os quais se relacionam com o sistema ecológico, provendo a sua existência através da extração, consumo, produção e comercialização de materiais e produtos provenientes do uso e manejo dos recursos existentes no sistema ambiental. A abordagem sistêmica aplicada à gestão socioambiental reconhece a interdependência de sub-sistemas (qualquer que seja a sua delimitação) produzidos pela interação entre seres humanos e natureza, caracterizados por alto grau de complexidade e incerteza.

A abordagem conceitual da resiliência reconhece que os sistemas socioecológicos são dinâmicos e complexos, com a possibilidade de múltiplos equilíbrios e mudanças não-lineares, onde vários caminhos são possíveis e difíceis de prever. Chama a atenção para a capacidade do sistema de se adaptar e mudar sem perder os elementos, estrutura e relações fundamentais que caracterizam seu regime de existência. O futuro é imprevisível e a resiliência trata da capacidade de um sistema de aprender, se auto-organizar e responder a perturbações.

Não se pode perder de vista o fato de que as paisagens socioecológicas globais e amazônicas, moldadas em maior ou menor grau pela ação humana, são ativamente produzidas por modelos econômicos de desenvolvimento inseridos num sistema de governança regido por regras formais. Historicamente, o espaço amazônico apresenta-se como um palco de conflitos e lutas de poder sobre o uso e aproveitamento de seus diversos potenciais econômicos, sejam eles florestais, hídricos, agrícolas, minerários, entre outros. A diversidade social amazônica se caracteriza por uma multiplicidade de interesses, culturas, línguas, e sistemas de conhecimento (epistemologias) entre atores que socialmente reproduzem seus modos de vida em interação com o ambiente. Em meio à esta diversidade de visões, poderes, interesses e práticas, produz-se uma fronteira Amazônica contestada, em movimento, onde indígenas, ribeirinhos, quilombolas, agricultores, pecuaristas, produtores florestais e moradores urbanos vivem suas vidas e procuram defender os seus interesses e direitos.

Governar os sistemas amazônicos subentende, portanto, gerir esta diversidade socioecológica e suas conexões locais e trans-escalares, reconhecendo as relações de poder, bem como as perdas e os ganhos de diferentes arranjos institucionais e decisões para diferentes atores. Neste cenário, a abordagem do desenvolvimento participativo reconhece a diversidade social e as relações de poder inerentes às sociedades humanas. Em tese, o desenvolvimento participativo preconiza o direito à participação, à emancipação e ao empoderamento de grupos sociais em processos de tomada de decisão, com base em suas realidades e formas de entender o mundo. Na pesquisa participativa, que pode envolver diversos níveis de participação dos grupos envolvidos, os participantes são considerados sujeitos da pesquisa (em contraposição à noção de informante ou "objeto" de pesquisa), e podem deter um menor ou maior controle sobre decisões relacionadas à pesquisa (como definição de objetivos, etapas, atividades e produtos da pesquisa).

A pergunta que motiva este dossiê é: Como uma abordagem sistêmica que incorpore o conceito de resiliência pode contribuir para a gestão socioambiental? No caminho de exploração desta pergunta, chegamos a outra pergunta chave: Quem deve avaliar o potencial de exploração e manejar a resiliência de um sistema socioecológico?

Neste dossiê, pretende-se contribuir para enfrentar o desafio da gestão socioambiental voltada à resiliência da socio-biodiversidade amazônica por meio de uma abordagem que integra conceitos e métodos de avaliação da resiliência com princípios, métodos e ferramentas pedagógicos da aprendizagem experiencial e da pesquisa participativa.

Os artigos que compõem esta coletânea apresentam resultados e reflexões de um grupo multi-disciplinar e multi-institucional de 23 profissionais - representativos de uma diversidade de áreas disciplinares, instituições e de experiências práticas na Amazônia - que participaram de um programa internacional de formação de lideranças e pesquisa colaborativa realizado no Mato Grosso, na Amazônia brasileira, entre 2010 e 2012. Esta iniciativa deu-se em continuidade a um curso de campo realizado na região de 
Alta Floresta em 2009, o qual gerou um grupo multidisciplinar que propôs a organização de um processo formativo que possibilitasse a realização de pesquisas e produções científicas institucionalizadas e compartilhadas com a comunidade local $^{3}$. Como resultado desta proposta, foi realizado um curso de pós-graduação Lato Sensu, intitulado "Gestão Colaborativa de Sistemas Socioecológicos na Amazônia Brasileira", complementado por um projeto de pesquisa, com o objetivo de analisar a dinâmica de uso da terra e gestão socioambiental no município de Cotriguaçu, no Mato Grosso. O processo foi coordenado pelo programa "Amazon Conservation Leadership Initiative - $A C L L^{2 "}$ da University of Florida e pela Universidade do Estado de Mato Grosso - UNEMAT, em parceria com o Instituto Centro de Vida - ICV e a Secretaria de Estado de Meio Ambiente do Mato Grosso - SEMA/MT. O curso e o projeto de pesquisa foram realizados de forma integrada e tiveram apoio financeiro da Gordon and Betty Moore Foundation dos Estados Unidos, e da Fundação de Amparo à Pesquisa do Estado de Mato Grosso (FAPEMAT).

O curso foi desenvolvido em quatro módulos presenciais complementados por atividades de intercâmbio, formação acadêmica, mídias sociais e aprendizagem à distância. O uso de ferramentas interativas, jogos, discussões e atividades de campo possibilitou a troca de experiências e a potencialização dos processos de aprendizagem, reflexão e aplicação dos conteúdos e métodos do curso (Figura 1). Os participantes tiveram a oportunidade de interagir com diversos atores sociais que tem a posse e fazem distintos usos da terra na região rural de Cotriguaçu, os quais são representativos de áreas de fronteira na Amazônia. Foram formados três grupos que realizaram estudos diagnósticos e levantaram elementos para a avaliação de resiliência para os três principais grupos sociais rurais em Cotriguaçu, considerado o sistema focal: (i) proprietários de terras médias e grandes que se dedicam a exploração madeireira e pecuária, (ii) comunidades indígenas do Povo Rikbaktsa, e (iii) agricultores familiares. Esses grupos de participantes trabalharam para entender a história, caracterizar os principais atributos e objetivos, e considerar cenários futuros para cada grupo social. Para a fase final da análise, os grupos do curso se reuniram para considerar as interações entre os diferentes grupos sociais, e como potenciais ações de gestão poderiam desencadear diferentes futuros para estes grupos, tanto individual como coletivamente, reconhecendo perdas, ganhos e relações de poder.
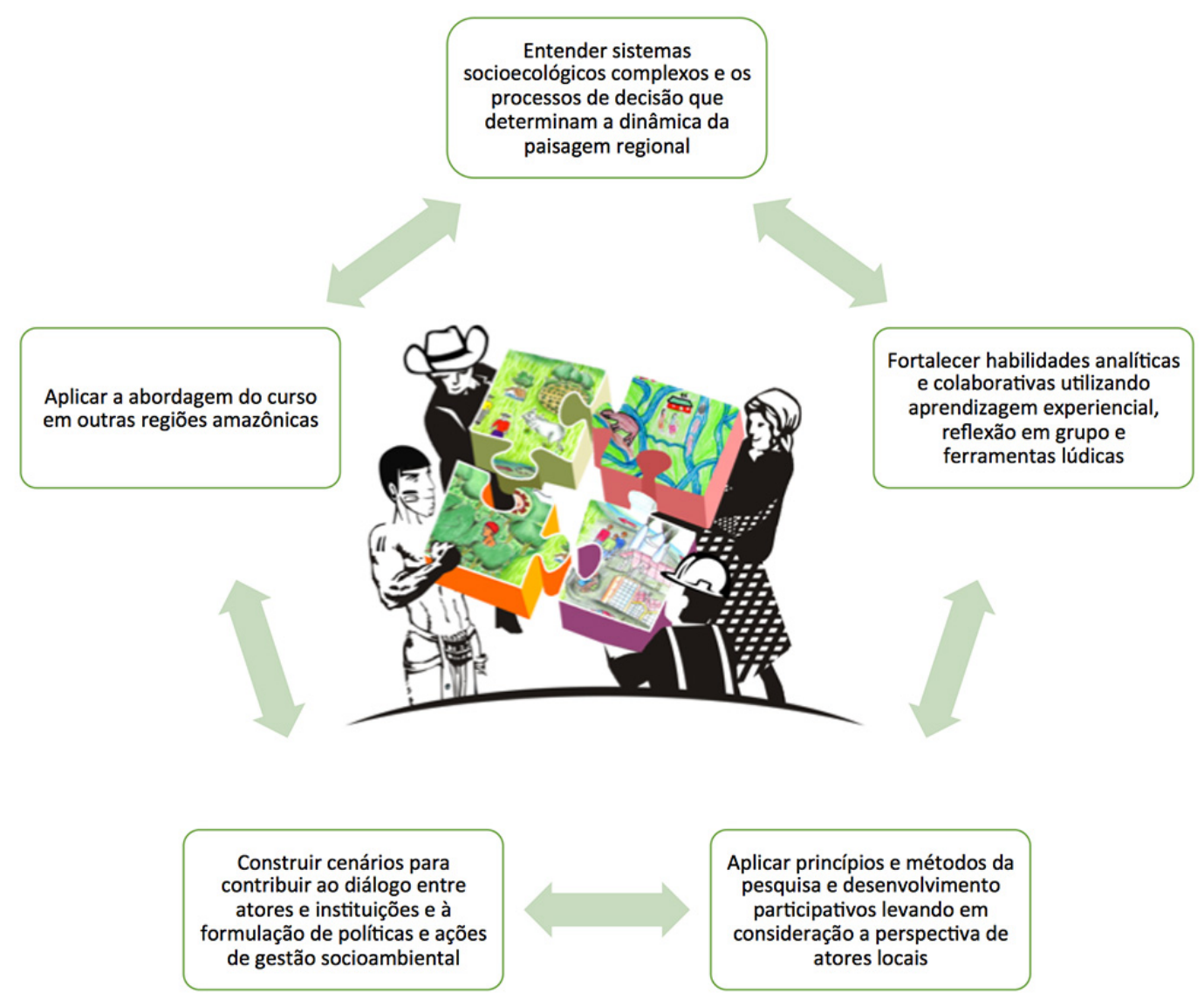

Figura 1 - Objetivos e arcabouço teórico-metodológico adotados no curso de pós-graduação Lato Sensu "Gestão Colaborativa de Sistemas Socioecológicos na Amazônia Brasileira." 
Em julho de 2012, com apoio de moradores e da prefeitura municipal de Cotriguaçu, o grupo de participantes do curso organizou um evento de restituição dos resultados e produtos do curso na Praça Municipal de Cotriguaçu. O evento mobilizou muitos dos residentes do município para interagir com os participantes, o que lhes permitiu conhecer a abordagem, as atividades, e os resultados atingidos nos dois anos de realização desta iniciativa (Figura 2).

A

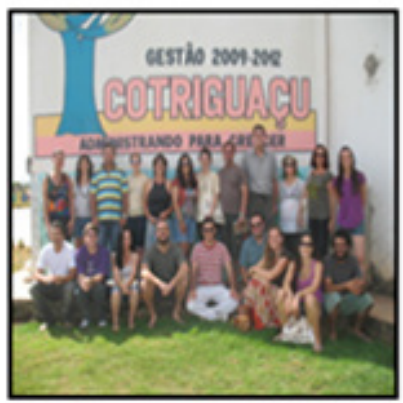

D

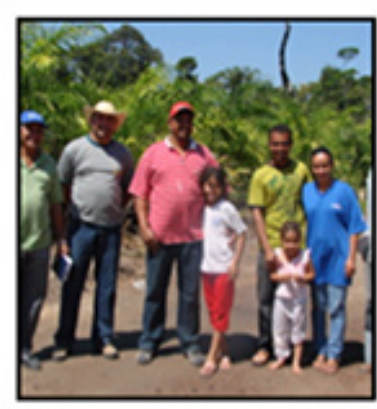

G

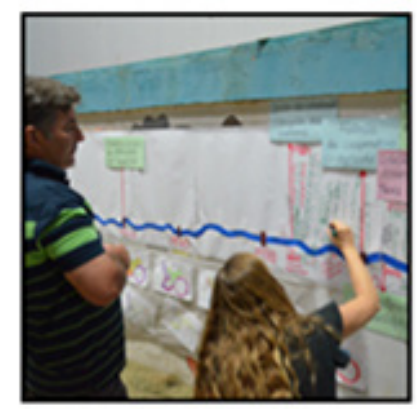

B

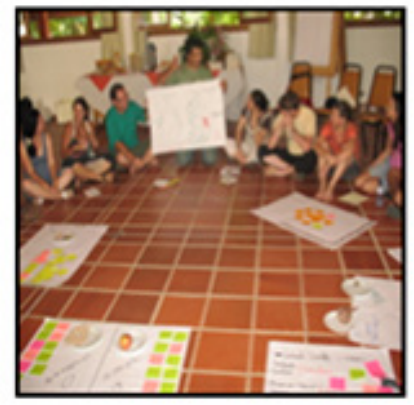

E

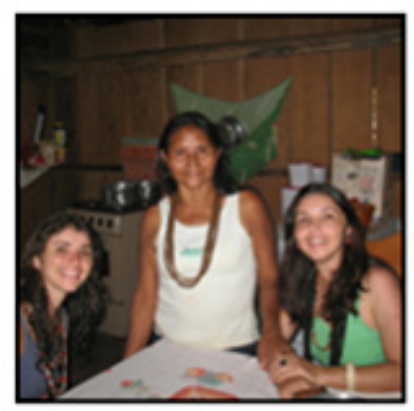

H

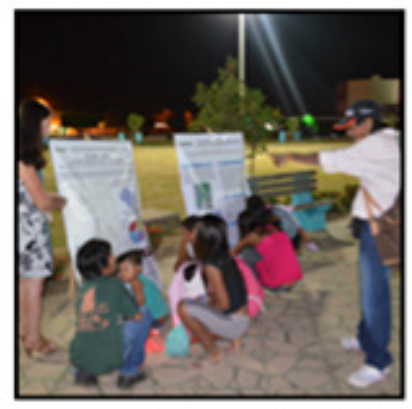

C

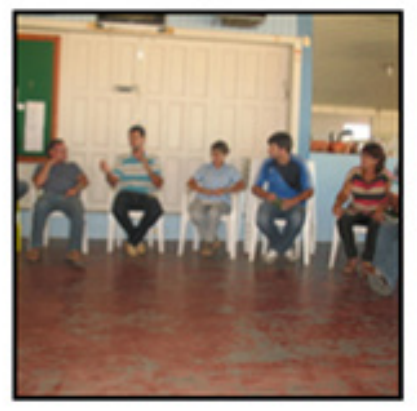

F

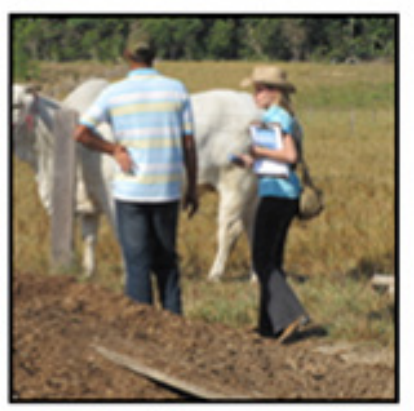

I

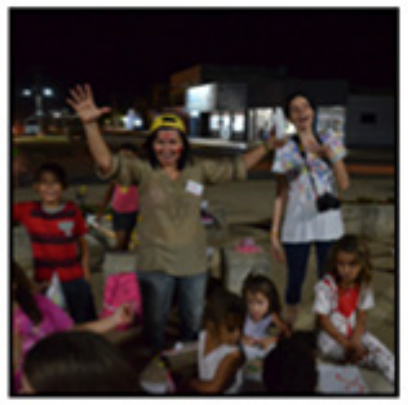

Figura 2 - Momentos de destaque do curso. A. Grupo do curso na praça municipal de Cotriguaçu. B. Ferramenta café Paulo Freire, utilizada para trabalhar temas geradores a partir da vivência dos participantes. C. Roda de conversa com gestores municipais. D-F. Interação com atores locais da agricultura familiar, povo indígena Rikbaktsa e produção pecuária. G-I. Evento de devolutiva realizado na Praça Municipal de Cotriguaçu. G. Linha do tempo interativa construída com moradores. H. Participação de representantes de comunidades Rikbaktsa. I. Atividades lúdicas realizadas com as crianças de Cotriguaçu. Fotos: A-C; E; G-I: Simone Athayde. D: Paula Bernasconi. F: Rosane Seluchinesk.

O presente dossiê é composto por sete artigos acadêmicos, um debate, uma entrevista, quatro resenhas de livros e, por fim, uma galeria de imagens. Os artigos congregam um conjunto de experiências aplicando teorias e métodos ligados à abordagem dos sistemas socioecológicos complexos, resiliência e aprendizagem colaborativa, avaliando a sua aplicabilidade para subsidiar processos de gestão socioambiental na Amazônia.

O dossiê inicia-se com o artigo introdutório de Robert Buschbacher e colaboradores, que apresenta a abordagem da avaliação de resiliência trabalhada ao longo do curso, ao tempo que sistematiza e 
discute os principais resultados da aplicação desta ferramenta analítica por grupos de participantes do curso, os quais trabalharam com três grupos de atores sociais locais. Na sequência, os participantes apresentam resultados do exercício de Avaliação de Resiliência em artigos de pesquisa, com foco nos proprietários de terras médias e grandes de Cotriguaçu (Paula Bernasconi e colaboradores), e em comunidades indígenas Rikbaktsa que habitam Cotriguaçu e outros territórios no noroeste do Mato Grosso (Juliana Almeida e colaboradores).

Além dos exercícios em grupo, cada participante do curso realizou um projeto individual aplicando conceitos de resiliência ao seu próprio trabalho, em Cotriguaçu ou em outras regiões da Amazônia brasileira. Alexandre Olival adaptou no seu artigo a metodologia de Avaliação de Resiliência como uma ferramenta para o planejamento estratégico e operacional do Instituto Ouro Verde (IOV), uma organização não-governamental que atua apoiando agricultores familiares no norte de Mato Grosso. Berenice Simão e Simone Athayde dedicaram o seu trabalho a avaliar a resiliência cultural de comunidades ribeirinhas tradicionais que sofreram deslocamento forçado devido à construção da barragem hidrelétrica de Jirau, em Rondônia. Finalmente, Thaissa Sobreiro analisou a dinâmica da pesca ornamental no município de Barcelos no Amazonas, focando na evolução histórica do sistema, suas reorganizações e resiliência.

Na conclusão do dossiê, Wendy-Lin Bartels e colaboradores apresentam uma reflexão crítica sobre o potencial e os desafios da aplicação do manual de avaliação da resiliência desenvolvido pelo grupo internacional Resilience Alliance ${ }^{3}$ para a gestão socioambiental de regiões socio-biodiversas e contestadas como as fronteiras agrícolas da Amazônia. Por meio de perguntas como: "Quem e como os diferentes usuários dos recursos podem participar efetivamente das decisões de gestão que afetam os seus modos de vida? e Como conciliar diferenças e objetivos normalmente contrastantes?", os autores deste artigo discutem questões fundamentais relacionadas ao entendimento e "gestão" da diversidade de epistemologias, interesses, e relações de poder representados da Amazônia.

Apresenta-se na sequência um debate, coordenado por Paula Bernasconi e Robert Buschbacher, envolvendo três profissionais participantes do curso: Elison Marcelo Schuster, Renato Farias e André Baby, ligados respectivamente ao setor privado (produção florestal e pecuária), a ONG Instituto Centro de Vida - ICV, e a Secretaria de Estado de Meio Ambiente do Mato Grosso - SEMA/MT. No debate, os profissionais apresentaram suas opiniões e perspectivas sobre a gestão socioambiental em áreas de fronteira na Amazônia, e foram unânimes ao reconhecer que ações de comando e controle só conseguem controlar o desmatamento quando acompanhadas por incentivos econômicos.

A entrevista com o Professor Lance Gunderson (membro fundador do grupo Resilience Alliance, co-editor do periódico Ecology and Society e professor da Emory University), conduzida por Simone Athayde, Robert Buschbacher e Paula Bernasconi, ilustra pontos importantes sobre o surgimento e aplicação do conceito de resiliência nos Estados Unidos e no mundo, incluindo as regiões do Parque Nacional dos Everglades e da bacia do rio Klamath nos EUA, a grande barreira de corais na Austrália, e o sistema de gestão de áreas protegidas na África do Sul.

Complementando os artigos, as quatro resenhas de livros publicados recentemente em português, espanhol e inglês são uma contribuição importante para o debate atual sobre o entendimento e a gestão da diversidade socioecológica na América Latina; os caminhos alternativos para o atual modelo de desenvolvimento baseado no capitalismo prevalente no mundo globalizado; e a sustentabilidade de serviços ecossistêmicos a partir de ações de fortalecimento da resiliência socioecológica. A primeira resenha, preparada por Adriano Castorino e Rosane Seluchinesk, discorre sobre o livro A Queda do Céu: Palavras de um xamã yanomami, escrito pelo líder indígena Davi Kopenawa (Yanomami) em conjunto com o antropólogo Bruce Albert. O texto, escrito a quatro mãos, trata da cosmologia do povo Yanomami, das suas formas de perceber e interagir com o mundo, e das relações de reciprocidade com a natureza. Há no texto uma série de passagens em que Davi explica como foi o seu processo de iniciação para se tornar um Xamã. Este livro se conecta e tem eco nas imagens oníricas apresentadas na Galeria de Imagens Vôos Xamânicos, no final deste dossiê. 
A segunda resenha, elaborada por Susan Paulson para o livro Degrowth: a vocabulary for a new era (traduzido como Decrescimento: um vocabulário para uma nova era), co-editado por Giacomo D’Alisa, Federico Demaria e Giorgos Kallis, é o primeiro livro em língua inglesa que apresenta a proposta do decrescimento de forma abrangente. A terceira resenha foi preparada por Walterlina Brasil para o livro Masculinidades en movimiento: transformación territorial y sistemas de género, da autora Susan Paulson. Esta obra integra questões de desenvolvimento rural, ecologia política e gênero, apresentando análises que relacionam mudanças ocorridas no uso do território com novos modelos de desenvolvimento econômico, e a distribuição do trabalho entre homens e mulheres.

A quarta resenha foi elaborada por Robert Buschbacher para o livro Principles for Building Resilience: Sustaining Ecosystem Services in Social-Ecological Systems, co-editado por Reinette Biggs, Maja Schlüter e Michael L. Schoon. O livro, um produto da rede Aliança de Jovens Pesquisadores para a Resiliência, em colaboração com pesquisadores sêniores da Resilience Alliance, traz temas e abordagens que se conectam de diferentes formas aos artigos deste dossiê. Os autores deste volume compartilham preocupações de equidade e o potencial de conflito e de captura de benefícios e recursos por elites, inerentes a soluções de perdas e ganhos (trade-offs) para manter resiliência de serviços ecossistêmicos. Estas incluem trocas inter-generacionais, compensações inter-escalares (local vs. mundial), e compensações entre diferentes usuários dentro de uma determinada escala.

Por fim, a galeria de imagens Voos Xamânicos: Uma Imagética da Diversidade Biocultural Amazônica, com imagens da etnoecóloga e artista plástica Ruth Albernaaz (designação artística) e texto de Simone Athayde e Ruth Albernaz-Silveira, foi adaptada da obra apresentada em exposição na Galeria do Sesc Arsenal, em Cuiabá - MT, de novembro a dezembro de 2014, com curadoria da Profá. Dra. Imara Pizzato Quadros. O trabalho nos convida a dialogar com outras formas de entendimento do mundo expressas em sistemas de conhecimento indígenas, nos quais arte e ciência se integram na relação imemorial com a natureza. As imagens propiciam uma imersão visual e onírica num universo de seres Amazônicos, com destaque para aves e pássaros xamãs, com pinceladas literárias do poeta pantaneiro Manoel de Barros. O tema central da galeria é a conexão com o Xamã ou Pajé, curador, feiticeiro ou benzedor - figura central entre os povos indígenas amazônicos, no ofício da cura e da comunicação com o mundo dos espíritos.

Gostaríamos de agradecer às pessoas e instituições que contribuíram para a realização desta iniciativa. Um agradecimento especial aos residentes de Cotriguaçu - povo indígena Rikbaktsa, agricultores familiares, pecuaristas, produtores florestais, e moradores urbanos - cuja hospitalidade e participação fizeram de nosso curso uma experiência valiosa. Nossos agradecimentos aos financiadores Gordon and Betty Moore Foundation e FAPEMAT pelo apoio, bem como às instituições Prefeitura Municipal de Cotriguaçu, Universidade do Estado de Mato Grosso, Instituto Centro de Vida, Secretaria de Estado do Meio Ambiente do Mato Grosso, Fazenda São Nicolau - ONF Brasil, Fundação Nacional do Índio - FUNAI, e Programa Tropical Conservation and Development -TCD da University of Florida.

\section{NOTAS}

${ }^{1}$ Uma descrição detalhada das abordagens e resultados encontrados nos dois cursos pode ser encontrada em: Athayde, S. F.; Buschbacher, R.; Bartels, W.; Seluchinesk, R. D. R. Aprendizagem colaborativa e gestão de sistemas sócioecológicos na Amazônia: experiências de construção de conhecimento entre academia e sociedade. Revista Brasileira de Pós-Graduação (RBPG), 10(21): 729-756, 2013.

${ }^{2}$ O programa ACLI é vinculado ao Tropical Conservation and Development Program - TCD, baseado no Center for Latin American Studies e à School of Forest Resources and Conservation na University of Florida. http://www.tcd. ufl.edu/ 